\title{
The advantages of disposable screen-printed biosensors in a bioelectronic tongue for the analysis of grapes
}

\author{
C. Medina-Plaza a , C. García-Hernández a, J.A. de Saja b, J.A. Fernández-Escudero c, \\ E. Barajas ${ }^{\mathrm{d}}$, G. Medrano ${ }^{\mathrm{e}}$, C. García-Cabezón ${ }^{\mathrm{f}}$, F. Martin-Pedrosa ${ }^{\mathrm{f}}$, \\ M.L. Rodriguez-Mendez ${ }^{\text {a, * }}$
}

a Department of Inorganic Chemistry, Engineers School, Universidad de Valladolid, 47011 Valladolid, Spain

b Department of Condensed Matter Physics, Faculty of Sciences, Universidad de Valladolid, 47011 Valladolid, Spain

c Estacion Enologica de Castilla y Leon, C/Santísimo Cristo, 26, 47490 Rueda, Valladolid, Spain

d ITACYL Avenida de Burgos, KM.118, Finca Zamadueñas, 47071 Valladolid, Spain

e $R \& D$ Department, Bodega Cooperativa de Cigales, C/Las Bodegas, s/n, 47270 Cigales, Valladolid, Spain

${ }^{\mathrm{f}}$ Department of Materials Science, Engineers School, Universidad de Valladolid, 47011 Valladolid, Spain

\section{A R T I C L E I N F O}

\section{Article history:}

Received 2 December 2014

Received in revised form

19 February 2015

Accepted 21 February 2015

Available online 28 February 2015

\section{Keywords:}

Electronic tongue

Must

Electron mediator

Biosensor

\begin{abstract}
A B S T R A C T
Disposable screen-printed sensors have been modified with enzymes and used to form a bioelectronic tongue dedicated to the discrimination between different grape varieties. The multisensory system combined serigraphied electrodes modified with carbon, platinum, gold, graphene, Prussian blue and nickel oxide nanoparticles (M-SPE) covered with glucose oxidase (M-GOX-SPE) or tyrosinase (M-TyrSPE).

The M-GOX-SPE and M-Tyr-SPE sensors produced a variety of responses due to the different behavior of the electron mediators of the six screen-printed materials used for the electro-catalysis of the glucose and phenols by means of glucose oxidase and tyrosinase.

This variety of responses, together with the capability of the sensors to detect glucose or phenols, allowed the bioelectronic tongue developed here to discriminate between the juices obtained from different varieties of grape.

Partial least-squares (PLS-1) multivariate calibration of electrochemical data has been successfully applied to the simultaneous determination of glucose and polyphenols in musts.

The discrimination capability shown by this array of cheap and single-use sensors was similar to that found in other complex bioelectronic tongues. The lower price, ease of use and portability of the modified screen-printed electrode system makes the bioelectronic tongue developed here an alternative tool that can be used in situ in the vineyard block.
\end{abstract}

() 2015 Elsevier Ltd. All rights reserved.

\section{Introduction}

The use of accurate techniques for the analysis of grapes is an important need in the wine sector. Glucose and polyphenols are among the analytes that need to be monitored and measured in order to guarantee the maturity of the grapes and the quality of the final product. In the laboratory, the assessment of grape maturity is performed by analyzing representative samples by standard

\footnotetext{
* Corresponding author. Department of Inorganic Chemistry, Escuela de Ingenierías Industriales, Paseo del Cauce, 59, 47011 Valladolid, Spain. Tel.: +34983 423540; fax: +34 983423310
}

E-mail address: mluz@eii.uva.es (M.L. Rodriguez-Mendez). analytical techniques such as spectroscopy, chromatography (to measure phenols) or refractometry (to measure the sugar content in ${ }^{\circ}$ Brix) (Blouin \& Guimberteau, 2000). In the last few years, a variety of sensors dedicated to the grape analysis have been developed. Most of them are fiber-optic fluorescence sensors that measure the levels of anthocyanins and chlorophyll (Ghozlen, Cerovic, Germain, Toutain, \& Latouche, 2010), or near-infrared optical sensors that can assess the sugar content ( $\left.{ }^{\circ} \mathrm{Brix}\right)$ (Herrera, Guesalaga, \& Agosin, 2003). Electrochemical glucose and polyphenol biosensors are a powerful alternative for monitoring such compounds due of their specificity, high sensitivity and short response time (Barroso, De los Santos Alvarez, Delerue-Matos, \& 
Oliveira., 2011; Carralero Sanz, Mena, González-Cortés, YáñezSedeño, \& Pingarrón, 2005; Wang, 2008).

Typical enzymatic electrodes, based on glucose oxidase (or tyrosinase), undergo several electrochemical steps which produce a measurable current that is linearly related to the glucose (or polyphenol) content. The active sites of glucose oxidase and phenoloxidases are not accessible. In order to improve the electron transfer, electron mediators must be included in the sensor (Barroso et al., 2011; Chaubey \& Malhotra, 2002; Ricci, Amine, Palleschi, \& Moscone, 2003; Wang, 2008). Nanotechnology has opened up new opportunities to obtain more efficient electron mediators, such as metal nanoparticles or nanocarbons (i.e. carbon nanotubes or graphene) (Carralero et al., 2005; Rahman, Ahammad, Jin, Ahn, \& Lee, 2010).

The analysis of complex mixtures such as wines will be improved by using the so-called electronic tongues, a new class of instruments that provide global information about the sample, instead of information about specific compounds. There have been a range of portable electronic tongues which have been produced based on a numerous core sensing technologies. including mass, optic, electrical or electrochemical transduction (Baldwin, Bai, Plotto, \& Dea, 2011; Haddi et al., 2014; Riul, Dantas, Miyazaki, \& Oliveira, 2010; Rodríguez-Méndez, Medina, De Saja, Apetrei, \& Muñoz, 2012; Sghaier, Barhoumi, Maaref, Siadat, \& JaffrezicRenault, 2009; Sliwinska, Wisniewska, Dymerski, Namiesnik, \& Wardencki, 2014; Smyth \& Cozzolino, 2013). Arrays of electrochemical sensors have been successfully used to discriminate between wines with different organoleptic characteristics (Apetrei et al., 2012; Cetó, Apetrei, Del Valle, \& Rodríguez-Méndez, 2014; Cetó et al., 2012; Gay et al., 2010; Gil-Sánchez et al., 2011; Gutiérrez, Moreno-Barón, Pividori, Alegret, \& Del Valle, 2010; Parra, Hernando, Rodríguez-Méndez, \& De Saja, 2004; RodríguezMéndez et al., 2014; Zeravik, Hlavacek, Lacina, \& Skládal, 2009) or grapes of different qualities (Gutiérrez et al., 2011; Moreno i Codinachs et al., 2008). It has also been established that arrays combining sensors and biosensors can be advantageous, as they bring together both the benefits of classical arrays of electrochemical sensors (which provide global information about the sample) with the specificity of the enzyme-substrate reaction typical of biosensors (Medina-Plaza, De Saja, \& Rodríguez-Méndez, 2014; Medina-Plaza et al., 2014; Moreno i Codinachs et al., 2008).

In spite of the good results obtained with electronic and bioelectronic tongues, the use of liquid electrochemical cells and heavy potentiostats, the high cost of the sensors and the need for periodic calibration all restrict their use to the laboratory. It would be desirable to develop multisensor systems using disposable electrodes that could be used in the vineyard block.

During recent years, the screen-printing (thick film) technology, applied to sensor and biosensor construction, has been considerably improved. Screen-printed electrodes (SPEs) are in fact simple to prepare, rapid and versatile, and this technology also appears to be the most economical. Their low cost makes possible to use them as disposable electrodes. SPE containing a serigraphied pseudoreference $\mathrm{Ag} / \mathrm{AgCl}$ electrode, a counter electrode and a working electrode in the same device, can be prepared using a variety of modifiers that include metals, metal oxides, nanocarbons, nanoparticles, phthalocyanines, conducting polymers, or prussian blue, among many others (Metters, Kadara, \& Banks, 2011). Moreover, enzymes can be immobilized on modified screen-printed electrodes to take advantage of the electron mediator properties of many of these materials for biosensing.

The aim of this work was to develop a bioelectronic tongue, based on an array of disposable screen-printed electrodes, dedicated to the analysis of grapes. For this purpose, six SPE sensors, modified with different materials (M-SPE) were selected, including (a) three classical electrode materials: graphite (C-SPE), platinum (Pt-SPE) and gold (Au-SPE) (b) two nanostructured electrocatalytic materials: graphene (GPH-SPE) and nickel oxide nanoparticles (NiONP-SPE) and (c) materials with well-known electron mediator properties and Prussian Blue (PB-SPE). Then, glucose oxidase (GOX) or Tyrosinase (Tyr) were immobilized on the surface of the M-SPE electrodes to obtain M-GOX-SPE and M-Tyr-SPE biosensors that could provide information about the glucose and phenol content respectively. After the optimization of the measurement conditions and the evaluation of the cross-selectivity of the sensors, the array was used to analyze musts prepared from different varieties of grapes. The discriminatory capability of the sensor array was investigated using Principal Component Analysis (PCA).

\section{Material and methods}

\subsection{Chemicals}

All chemicals and solvents were purchased from Sigma-Aldrich. Solvents were of reagent grade and used as supplied. Deionized water was obtained from a Millipore purifier, with a resistivity of $18.2 \mathrm{M} \Omega / \mathrm{cm}$.

Glucose oxidase (GOX), from Aspergillus niger, type VII (activity plus $0.001 \mathrm{~kat} / \mathrm{mg}$ ), and Tyrosinase (Tyr), from Mushroom (activity of $6.10^{-5} \mathrm{~kat} / \mathrm{mg}$ ), were purchased from Sigma Chemical Co. (USA).

\subsection{Grape samples}

Samples of five varieties of red grapes (Tempranillo, Garnacha, Cabernet-Sauvignon, Prieto Picudo and Mencía) were harvested in September 2013. For each variety, berries were collected in the vineyards of the 'Bodega Cooperativa de Cigales' and of the 'Instituto Tecnologico Agrario de Castilla y León', both located in the Valladolid area of Castilla y León (Spain). To obtain the musts, 200 berries were introduced in a plastic bag and crushed for $1 \mathrm{~min}$ manually. For each variety of grape, this process was carried out by septuplicate, giving a total of 35 samples. The musts were analyzed after separation from the seeds and the peel. The Oenological Center of Castilla y León carried out the chemical analysis of the grapes following international regulations (OIV, 2013). Parameters analyzed included the chemical indicators of the sugar content ( ${ }^{\circ}$ Brix) and of the polyphenolic content (Total Polyphenol Index: TPI). Total acidity (expressed as $\mathrm{g} / \mathrm{L}$ of tartaric acid) and $\mathrm{pH}$ were also analyzed, due to the influence of the $\mathrm{pH}$ and the ionic strength in the enzymatic activity. The results are collected in Table 1.

\subsection{Screen-printed electrodes and biosensors}

Six screen-printed electrodes modified with different materials (M-SPE), purchased from Dropsens (www.dropsens.com), were used as substrates for the deposition of enzymes. Each sensor device contained a serigraphied pseudo-reference $\mathrm{Ag} / \mathrm{AgCl}$ electrode (RE), a counter electrode (C or Pt) (CE) and a working electrode. As

Table 1

Results of the chemical analysis of grapes carried out by traditional chemical methods (seven replicas).

\begin{tabular}{lllll}
\hline Grape & ${ }^{\circ}$ Brix & $\mathrm{pH}$ & $\begin{array}{l}\text { Total acidity } \\
(\mathrm{g} / \mathrm{L})\end{array}$ & $\begin{array}{l}\text { Total polyphenol } \\
\text { index }\end{array}$ \\
\hline Prieto Picudo & $23 \pm 2$ & $3.61 \pm 0.08$ & $4.8 \pm 0.2$ & $15 \pm 3$ \\
Mencia & $21 \pm 2$ & $4.18 \pm 0.08$ & $4.3 \pm 0.2$ & $13 \pm 3$ \\
Tempranillo & $24 \pm 2$ & $4.20 \pm 0.08$ & $4.1 \pm 0.2$ & $21 \pm 4$ \\
Cabernet-Sauvignon & $23 \pm 2$ & $3.65 \pm 0.08$ & $4.1 \pm 0.2$ & $16 \pm 3$ \\
Garnacha & $23 \pm 2$ & $3.39 \pm 0.08$ & $4.4 \pm 0.2$ & $15 \pm 3$ \\
\hline
\end{tabular}


Table 2

List of the screen printed electrodes used in the multisensor system and acronyms used in this work.

\begin{tabular}{|c|c|c|c|}
\hline \multirow[t]{2}{*}{ Dropsens reference } & Sensors without enzyme & Sensors containing glucose-oxidase & Sensors containing tyrosinase \\
\hline & Modifier/acronym & Modifier/acronym & Modifier/acronym \\
\hline DRP-110 & Carbon/C-SPE & Carbon/C-GOX-SPE & Carbon/C-Tyr-SPE \\
\hline DRP-550 & Platinum/Pt-SPE & Platinum/Pt-GOX-SPE & Platinum/Pt-Tyr-SPE \\
\hline DRP-250AT & Gold/Au-SPE & Gold/Au-GOX-SPE & Gold/Au-Tyr-SPE \\
\hline DRP-110GPH & Graphene/GPH-SPE & Graphene/GPH-GOX-SPE & Graphene/GPH-Tyr-SPE \\
\hline DRP-110NI & NiO Nanoparticles/NiONP-SPE & NiO Nanoparticles/NiONP-GOX-SPE & NiO Nanoparticles/NiONP-Tyr-SPE \\
\hline DRP-710 & Prussian Blue/PB-SPE & Prussian Blue/PB-GOX-SPE & Prussian Blue/PB-Tyr-SPE \\
\hline
\end{tabular}

observed in Table 2, the modifiers covering the working electrode of the M-SPE devices included carbon (C), platinum (Pt), gold ( $\mathrm{Au}$ ), graphene $(\mathrm{GPH})$, nickel oxide nanoparticles (NiONP) and Prussian blue (PB) (Table 3).

The six screen-printed electrodes were modified with Glucose oxidase (GOX) or Tyrosinase (Tyr), using the drop casting technique followed by cross-linking. For this purpose, $10 \mu \mathrm{l}$ of a $70 \mathrm{~g} / \mathrm{L}$ solution of the corresponding enzyme (tyrosinase or glucose oxidase) in $0.01 \mathrm{~mol} / \mathrm{L}$ phosphate buffer $(\mathrm{pH}$ 7.0) were placed onto the working electrode surface. After drying at room temperature for $30 \mathrm{~min}$, cross linking was carried out by exposing the modified working electrode to glutaraldehyde vapors (20 g/L, water solution) for $20 \mathrm{~min}$. Throughout the process, CE and RE were protected with a mask to avoid contamination by the enzyme or the glutaraldehyde. Using this method, six GOX-based (M-GOX-SPE) and six Tyr-based (M-Tyr-SPE) biosensors were prepared (Table 2).

\subsection{Electrochemical measurements}

Electrochemical measurements were carried out in a portable (weight under $1 \mathrm{~g}$ ) potentiostat $\mu$ STAT 400 (from Dropsens). Cyclic voltammograms were registered from $-0.6 \mathrm{~V}$ to $+1.0 \mathrm{~V}$ (the scan started at $0 \mathrm{~V}$ ), at a sweep rate of $0.05 \mathrm{~V} / \mathrm{s}$. The applied potential was measured versus the screen-printed internal silver pseudoreference electrode. Sensors were immersed in the solution and polarized sequentially. The electrochemical experiments were performed at a controlled temperature of $21^{\circ} \mathrm{C}$. After each measurement, sensors were discarded and replaced by a new sensor and wines were measured randomly.

\subsection{Statistical analysis}

Curves were pre-processed through the adaptation to our case of a data reduction technique based on predefined response "bellshaped-windowing" curves called "kernels" (Parra et al., 2004). It is based on a compression method (Gutiérrez-Osuna \& Nagle, 1999). The voltammogram curve is multiplied by 10 smooth, bell-shaped windowing functions defined as

$$
K_{i}\left(V_{j}\right)=\frac{1}{1+\left(\frac{V_{j}-c_{i}}{a_{i}}\right)^{2 b_{i}}}
$$

where $a_{i}, b_{i}$ and $c_{i}$ define the width, shape and center of the different windowing functions $\mathrm{K}_{\mathrm{i}}$. Subsequently, data were integrated with respect to voltage. After compression, each voltammogram has been reduced to a vector of 10 variables.

Using this method, ten variables per curve were obtained which were used as the input data source for statistical analysis. In previous works, it has been demonstrate that this method provides similar results to other pre-treatment processes such as the wavelet transformation (WT) (Cetó et al., 2014) or using genetic algorithms (Prieto et al., 2013).

A non-supervised multivariate method, the Principal Component Analysis (PCA), was used to analyze the voltammetric curves and to evaluate the discrimination capability of the system. The data entry was a matrix with the samples in the rows. As we registered seven repetitions per sample, 7 rows per sample (one per repetition) were introduced in the matrix. Sensors were inserted in the colons ( 10 colons per sensor because 10 kernels were calculated per sensor).

Partial Least Squares (PLS-1) regression models were built to predict the phenolic and the sugar content of the musts.

All computations, and the chemometric analysis, were carried out using the software Matlab v5.3 (The Mathworks Inc., Natick, MA, USA) and Unscrambler (Camo).

\section{Results}

\subsection{Measures in glucose and in catechol}

As a preliminary experiment, the electrochemical response of screen-printed sensors immersed in $0.01 \mathrm{~mol} / \mathrm{L}$ phosphate buffer was tested. As expected, C-SPE, GPH-SPE and NiONP-SPE sensors did not show any redox peak within the studied range. Pt-SPE and Au-SPE showed the usual peaks related to the oxidation/reduction of water and those associated with the adsorption of oxidized species to the surface. Finally, PB-SPE showed the redox peaks of the hexacyanoferrate ion (To Thi Kim, Gabrielli, Perrot, GarciaJareno, \& Vicente, 2012).

The effect of the electrode modification with GOX on the oxidation of glucose at the SPE surface, and the role of the six materials used as electron mediators, was evaluated by performing voltammetric experiments. Fig. 1 compares the cyclic voltammograms of the M-SPE and M-GOX-SPE electrodes in the presence of $0.01 \mathrm{~mol} / \mathrm{L}$ glucose in phosphate buffer.

The responses of C-SPE, Pt-SPE and Au-SPE were similar to those of the electrodes modified with the enzyme (C-GOX-SPE, Pt-GOXSPE and Au-GOX-SPE). The only noticeable differences were small increases in the background currents in M-GOX-SPE, and a slight

Table 3

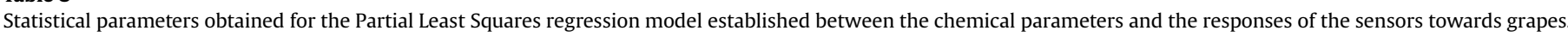

\begin{tabular}{|c|c|c|c|c|c|}
\hline Parameter & $\begin{array}{l}\text { Squared correlation } \\
\text { coefficient in calibration }\left(\mathrm{R}^{2} \mathrm{C}\right)\end{array}$ & $\begin{array}{l}\text { Root Mean Square } \\
\text { Error of Calibration } \\
\text { (RMSEC) }\end{array}$ & $\begin{array}{l}\text { Squared correlation } \\
\text { coefficient in prediction }\left(\mathrm{R}_{\mathrm{P}}^{2}\right)\end{array}$ & $\begin{array}{l}\text { Root Mean Square Error } \\
\text { of Prediction (RMSEP) }\end{array}$ & Latent variables \\
\hline Brix & 0.9565 & 5.4211 & 0.9432 & 6.1827 & 3 \\
\hline Total polyphenol index & 0.9821 & 0.4932 & 0.9127 & 1.2976 & 5 \\
\hline
\end{tabular}



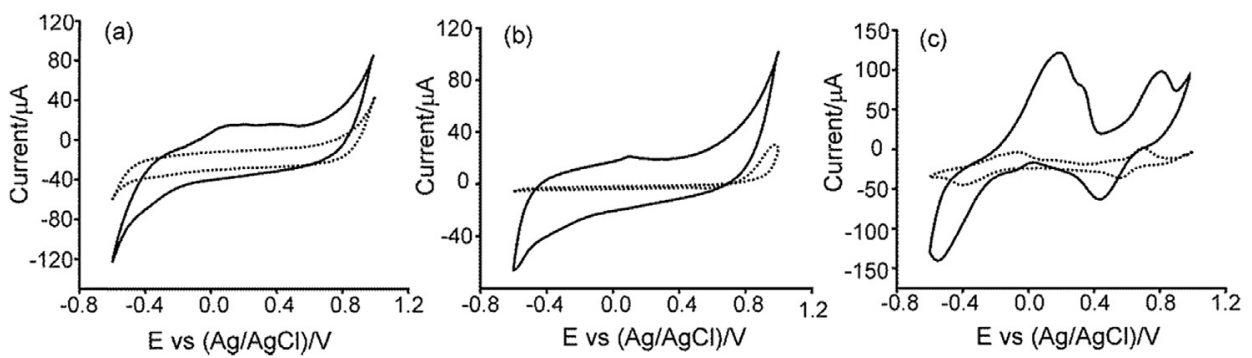

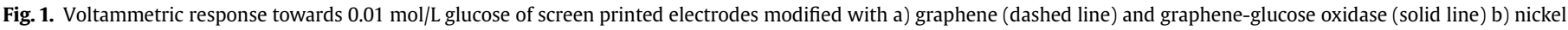

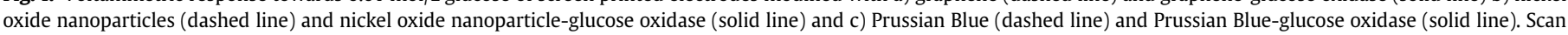
rate $0.05 \mathrm{~V} / \mathrm{s}$. Supporting electrolyte: $0.01 \mathrm{~mol} / \mathrm{L}$ phosphate buffer $(\mathrm{pH} 7.0)$.

increase in the current intensity at potentials related to the electrochemical reactivity of $\mathrm{H}_{2} \mathrm{O}_{2}$ (at $-0.4 \mathrm{~V}$ and lower). The curves obtained with GPH-GOX-SPE, NiONP-GOX-SPE and PB-GOX-SPE showed a clear increase in the current intensities of the whole curves, demonstrating that graphene, nickel oxide nanoparticles and Prussian blue were excellent electron mediators. It is important to remark that Prussian blue was particularly effective in enhancing the sensitivity towards glucose. The good electro-catalytic activity of such modifiers was in good agreement with results described in the literature using other types of electrodes (Rahman et al., 2010; To Thi Kim et al., 2012; Valentini, Carbone, \& Palleschi, 2013).

The electrochemical response of the M-SPE and M-Tyr-SPE sensors towards phenols was evaluated by registering the cyclic voltammograms in a $0.01 \mathrm{~mol} / \mathrm{L}$ catechol solution (in $0.01 \mathrm{~mol} / \mathrm{L}$ phosphate buffer $\mathrm{pH}$ 7.0). In all M-SPE sensors, the oxidation of catechol to the quinoid form was observed at ca. $0.8 \mathrm{~V}$. This peak was accompanied by a peak at ca. $-0.2 \mathrm{~V}$, produced by the reverse reaction (Matemadombo, Apetrei, Nyokong, Rodríguez-Méndez, \& De Saja, 2012). Only small differences in the position and intensities of the peaks were observed form one electrode to another. According to this, none of the modifiers tested showed a particularly intense electro-catalytic effect towards catechol.

When electrodes were covered with tyrosinase, the electrochemical response of C-Tyr-SPE, Pt-Tyr-SPE and Au-Tyr-SPE were almost identical to the responses observed in the absence of tyrosinase. In contrast, GPH-Tyr-SPE, NiO-Tyr-SPE and PB-Tyr-SPE, all produced a shift in the anodic peak to lower values (from $0.8 \mathrm{~V}$ to $0.65 \mathrm{~V}$ ). This effect was accompanied by a moderate increase in the intensity of the cathodic peak at $-0.2 \mathrm{~V}$ due to the reduction of the quinone derivative formed as a result of the enzymatic oxidation of catechol by the immobilized tyrosinase (Fig. 2).

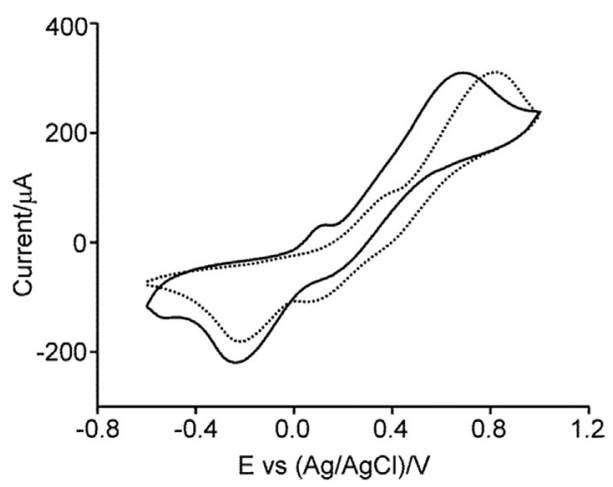

Fig. 2. Cyclic voltammograms registered using screen printed electrodes modified with nickel oxide nanoparticles (dashed line) and nickel oxide nanoparticlestyrosinase (solid line) immersed in $0.01 \mathrm{~mol} / \mathrm{L}$ catechol. Scan rate $0.05 \mathrm{~V} / \mathrm{s}$. Supporting electrolyte: $0.01 \mathrm{~mol} / \mathrm{L}$ phosphate buffer $(\mathrm{pH} 7.0)$.

\subsection{Measures in musts. Optimization of the experimental conditions}

As will be shown below, voltammograms registered by immersing the SPE electrodes in musts showed rich voltammograms where peaks produced by components with redox activity present in the sample (i.e. phenols, or sugars) and peaks related to the electrode modifier (i.e. Prussian blue) could be observed.

Unfortunately, preliminary experiments using M-SPE sensors demonstrated that the direct analysis of non-diluted musts produced irreproducible responses (the fact that the first cycle is always different from the rest has to be taken into account because, during this cycle, a flux of ions diffusing inside/outside the film is established, which is why this cycle was always discarded). Upon successive cycling, a progressive decrease in the intensity of the signals was observed, with a coefficient of variation (CV) always higher than $30 \%$ (Fig. 3a). This was due to the existence of proteins in suspension or the presence of other materials, such as sugars, that could adhere to the electrode surface. Moreover, the direct contact between the solution and the pseudo reference $\mathrm{Ag} / \mathrm{AgCl}$ electrode could also be a source of irreproducibility.

A 1:2 dilution with water or with $0.01 \mathrm{~mol} / \mathrm{L}$ phosphate buffer drastically improved the repeatability, and the CVs were within the range of $8-16 \%$ in M-SPE sensors not modified with enzymes (Fig. 3b). The variability was lower than $10 \%$ in sensors covered with enzymes.

In most of the cases, a 1:2 dilution with $0.01 \mathrm{~mol} / \mathrm{L}$ phosphate buffer produced voltammograms with a higher intensity than dilution with water. However, the degree of amplification was dependent on the nature of the electrode. This is illustrated in Fig. 4, where a clear increase in the intensity was observed when using GPH-Tyr-SPE; whereas the amplification was almost negligible when using a NiONP-Tyr-SPE electrode. The reasons for the amplification are not clear, but this phenomenon could be related to the increase in the $\mathrm{pH}$ produced by the phosphate buffer.

Regarding the lifetime, it is worth mentioning that M-SPE electrodes decreased their intensity smoothly over 50 cycles. In contrast, M-GOX-SPE or M-Tyr-SPE decreased their intensity progressively over ca. 10-15 cycles. After the 10-15th cycle, erratic behavior was observed and these electrodes should be discarded. According to this, each sensor was used to analyze one must sample.

Once the experimental conditions had been established, the array of sensors was used to analyze musts of five different grape varieties.

As mentioned in previous paragraphs, the sensors developed here provide complex voltammetric responses. The reason is that voltammetric curves take into account the electro-active compounds present in wines or musts (e.g. polyphenols or sugars). In addition, the electro-catalytic material facilitates the oxidation of 

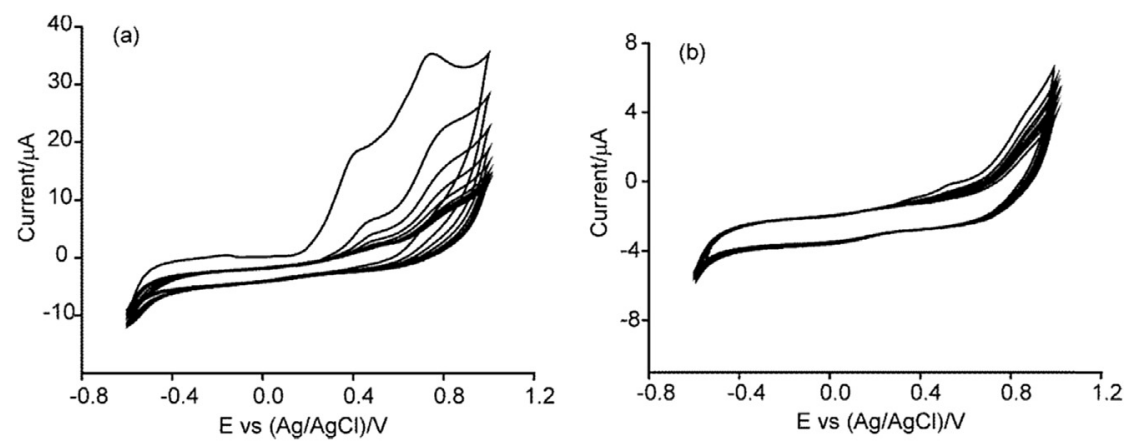

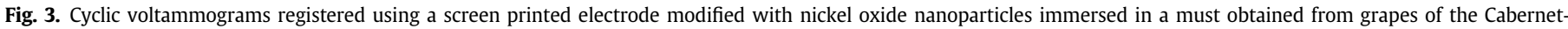
Sauvignon variety (a) undiluted sample and (b) must diluted 1:2 in $0.01 \mathrm{~mol} / \mathrm{L}$ phosphate buffer. Images correspond to 10 successive cycles.
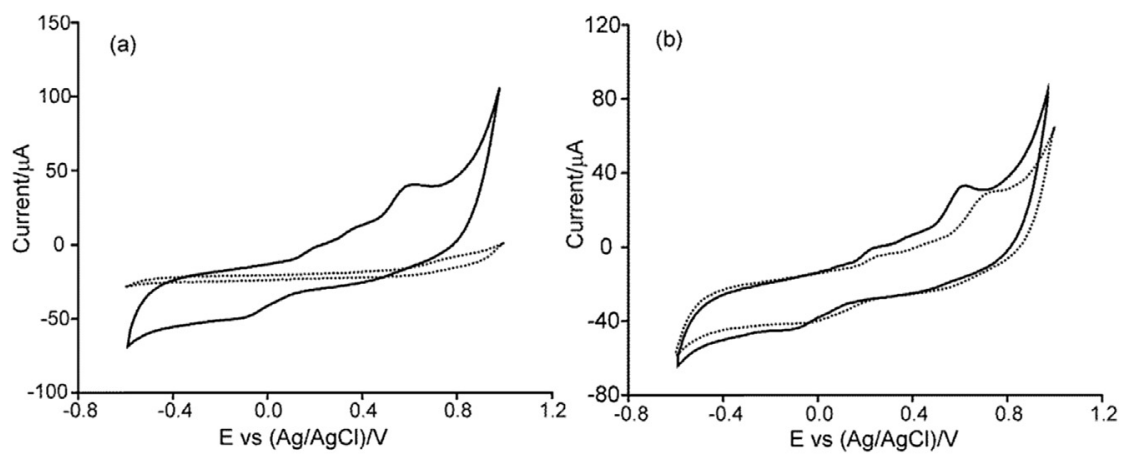

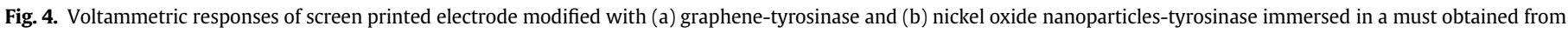
grapes of the Tempranillo variety. Dashed lines correspond to must diluted 1:2 in water and solid lines to musts diluted in 0.01 mol/L. phosphate buffer.

certain compounds such as phenols. These interactions increase the cross-selectivity of the sensors, thus improving the performance of the array. Finally, the modification with enzymes and the enzymeelectron mediator interaction introduce certain selectivity to the array.

For these reasons, each electrode showed a distinct response towards the musts analyzed, depending on the chemical composition of the working electrode. In all the M-SPE sensors, the oxidation peak of phenols present in the musts was observed to be within the $0.5-0.8 \mathrm{~V}$ region as a broad anodic wave (Fig. 5). Different intensities were observed depending on the nature of the working electrode.

When the electrodes were covered with GOX (Fig. 5 solid lines), a clear increase in the intensity of the curves was observed when the electron mediator was NiONP or PB, confirming the excellent electron mediator properties of nickel oxide nanoparticles and
Prussian blue for GOX. The electro-catalytic effect was not observed in C-GOX-SPE, Pt-GOX-SPE, Au-GOX-SPE or GPH-GOX-SPE.

The NiONP-Tyr-SPE and PB-Tyr-SPE biosensors also furnished substantially larger signals than the NiONP-SPE or PB-SPE analogs, reflecting the electro-catalytic activity of NiONP and PB for Tyrosinase (Fig. 6). This enhancement was particularly noticeable in the case of the NiONP electrodes, demonstrating the excellent characteristics of $\mathrm{NiO}$ nanoparticles as electrode material in the construction of electro-chemical enzyme sensors for the detection of phenolic compounds.

Obviously, the voltammetric responses depend on the chemical composition of the studied solution. As each grape has his own content in sugars and phenols, the same sensor provides different responses towards different grapes. This is illustrated in Fig. 7, where the response of the PB-Tyr-SPE immersed in different musts is shown.
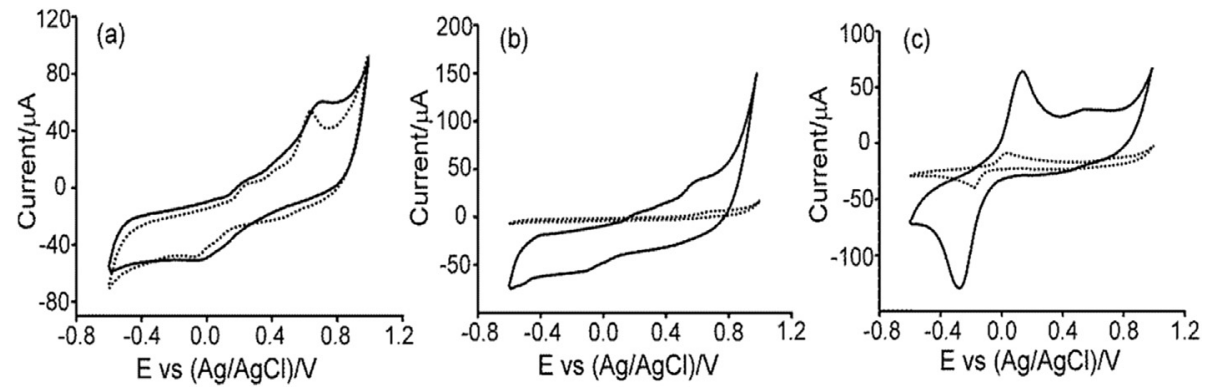

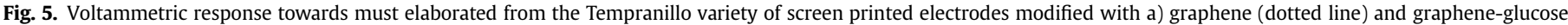

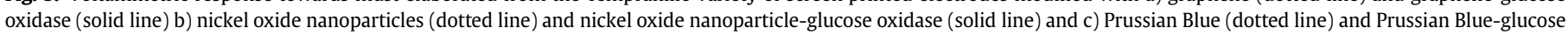
oxidase (solid line). Scan rate $0.05 \mathrm{~V} / \mathrm{s}$. Supporting electrolyte: $0.01 \mathrm{~mol} / \mathrm{L}$ phosphate buffer $(\mathrm{pH} 7.0$ ). 

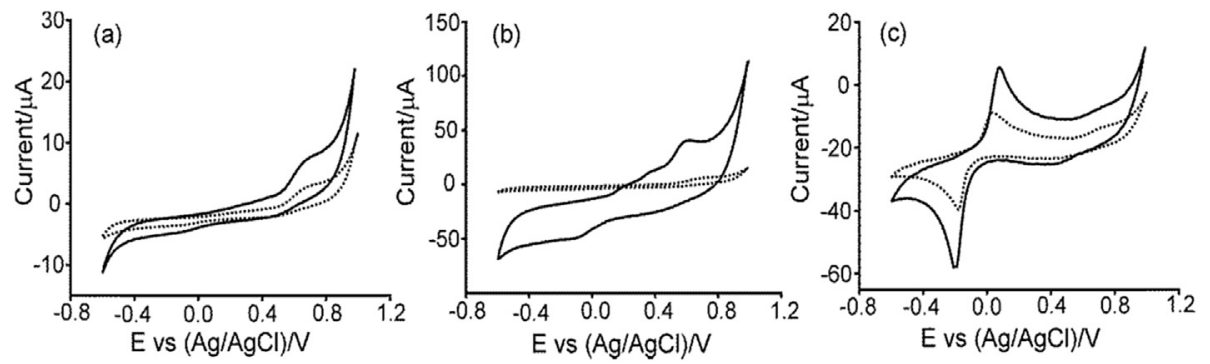

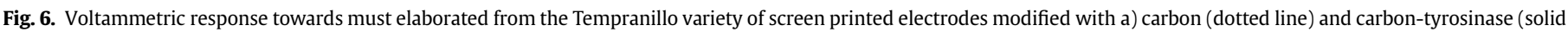

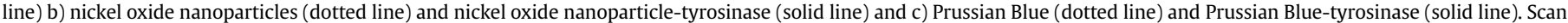
rate $0.05 \mathrm{~V} / \mathrm{s}$. Supporting electrolyte: $0.01 \mathrm{~mol} / \mathrm{L}$ phosphate buffer $(\mathrm{pH} 7.0)$.

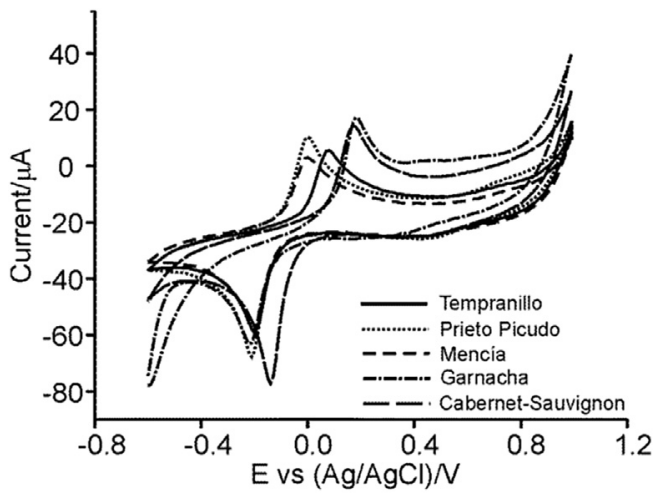

Fig. 7. Cyclic voltammograms registered using a screen printed electrode modified with Prussian Blue-tyrosinase immersed in five musts prepared from different grape varieties.

\subsection{Bioelectronic tongue. Statistical analysis}

As demonstrated in the previous section, the screen-printed electrodes were sensitive to the chemical compounds present in musts. In addition, the chosen electron mediators showed different electro-catalytic behaviors. According to these results, the electrodes provided distinct responses when immersed in different musts, showing an important degree of cross-selectivity.

Principal Component Analysis (PCA) was performed using the signals obtained separately from the arrays of M-SPE (Fig. 8a), MGOX-SPE (Fig. 8b), and M-Tyr-SPE electrodes (Fig. 8c). Notice that each array is formed by 6 sensors (number of variables in each case is 6 sensors $\mathrm{x} 10$ kernels).

As observed in the figure, the third principal component also brings an important amount of information. This result is usually found in voltammetric systems (Apetrei, 2012; Cetó et al., 2014; Prieto et al., 2013) and is the consequence of the large amount of information contained in these complex curves.

When using M-SPE sensors, the clusters corresponding to the five studied musts (seven replicas per must) were clearly overlapped and it was not possible to discriminate between them (Fig. 8a). The variation coefficients of the M-SPE sensors, calculated in Section 3.2, are responsible for the large dispersion. On the other hand, the arrays formed by the M-GOX-SPE or the M-Tyr-SPE sensors lead to a certain improvement of the discrimination. The most relevant aspect was that the positions of the clusters were related to the sugar and phenolic content of the musts analyzed. For instance, must prepared from the Tempranillo grape (which showed the highest sugar content as indicated by the ${ }^{\circ}$ Brix) was located on the left of Fig. 8b, whereas must prepared from Mencía grapes, with the lowest sugar content, was located on the right of
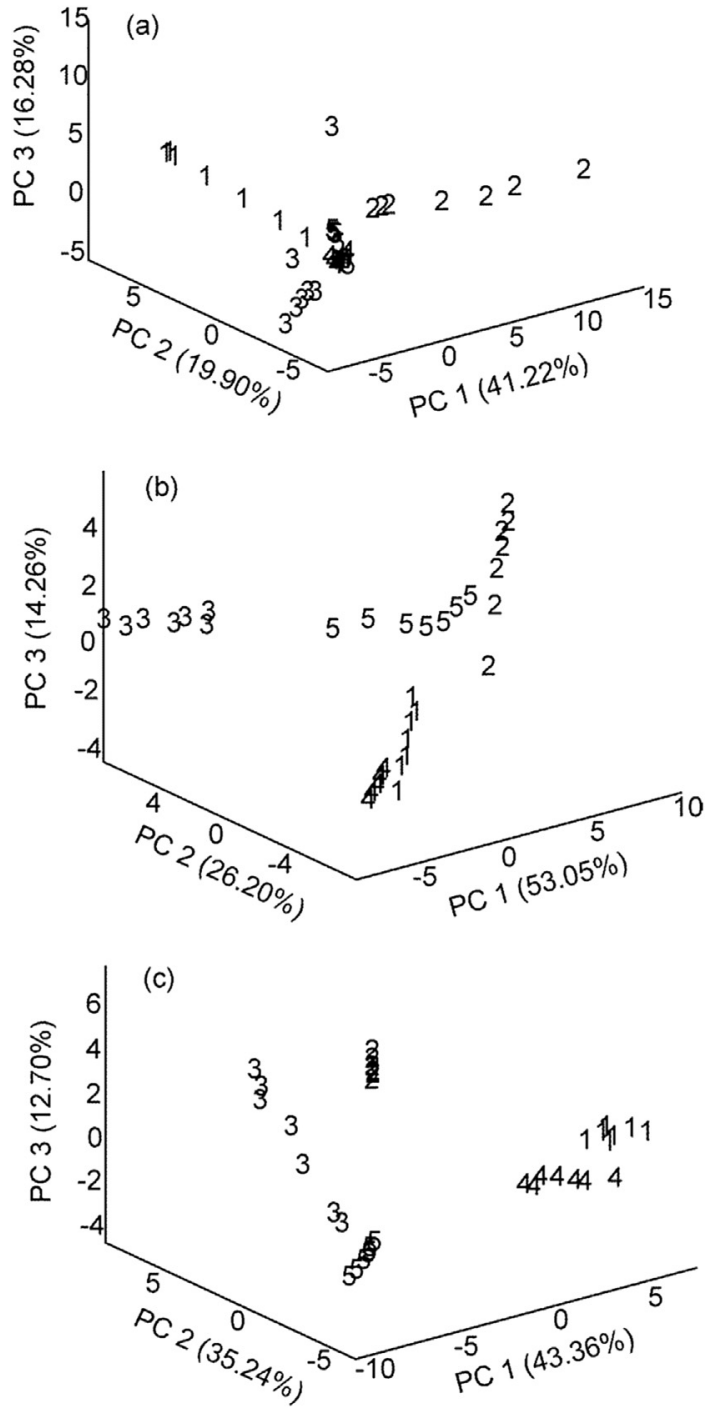

Fig. 8. Principal Component Analysis scores plot using arrays of (a) screen printed electrodes; (b) screen printed electrodes modified with glucose oxidase and (c) screen printed electrodes modified with tyrosinase. Must samples are 1: Prieto Picudo; 2: Mencía; 3: Tempranillo; 4: Cabernet-Sauvignon; and 5: Garnacha.

the figure. The discrimination using the array of M-Tyr-SPE was not so efficient, but some general trends could be observed (Fig. 8c). Must obtained from Tempranillo, Cabernet-sauvignon, Prieto Picudo and Garnacha grapes, which have the highest IPT values, tend to appear in the region corresponding to a negative PC2. Musts 

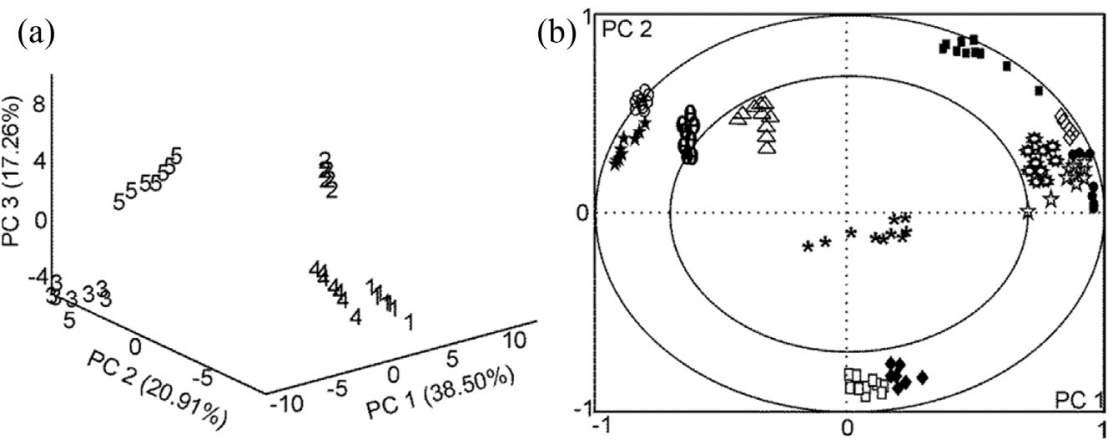

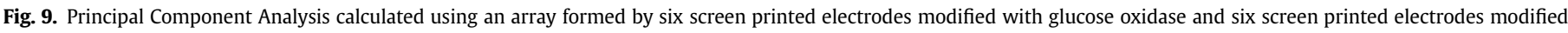

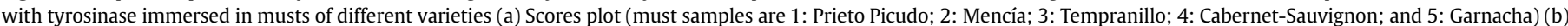

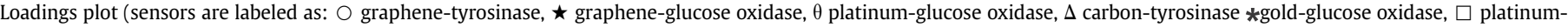

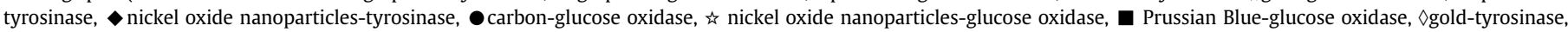
Prusian Blue-tyrosinase).

from the Mencía variety, with the lowest IPT, tend to appear in the positive area of PC2.

After testing the arrays separately, the multisensor systems were merged to obtain an array that can discriminate musts according to the sugar and phenolic content simultaneously. The combination of M-GOX-SPE and M-Tyr-SPE considerably improved the discrimination capability. This is illustrated in Fig. 9a, where the scores plot, calculated when using the combined biosensor array, is shown. As observed, using the mixed array, a clear separation between the five musts studied was attained. The positions of the clusters are related to the sugar and phenolic content. For instance, the Tempranillo variety, which has the higher ${ }^{\circ}$ Brix and IPT, appears apart from the rest in the negative region of $\mathrm{PC} 1$ and the positive region of PC2. Prieto Picudo, Cabernet-Sauvignon and Garnacha, which have intermediate values of ${ }^{\circ}$ Brix and IPT, appear in the middle of PC1, while Mencía, with the lowest values, appears in the negative region of PC1. It has to be noted that the cluster of the Garnacha variety (which has similar values of IPT and ${ }^{\circ}$ Brix to Prieto Picudo and Cabernet-Sauvignon) appears well separated from the cluster corresponding to the other two varieties, instead of being located in the same region. This can be attributed to the low $\mathrm{pH}$ values of the must obtained from this grape, which can cause important differences in the enzymatic activity. It is also worth mentioning that including the set of M-SPE sensors in the array did not significantly improve the discrimination capability of the system.

Fig. 9b shows the loading plots obtained by using the hybrid array formed by six M-GOX-SPE and six M-Tyr-SPE sensors (twelve sensors $\times 10$ kernels per sensor). Similar loadings indicate redundancy and collinearity in the response matrix. As observed in the figure, the biosensors have different loadings, indicating that they provide complementary information.

The system developed showed almost the same discrimination capability as other arrays based on more complex sensors with a high degree of cross-selectivity.

The PLS model was developed in the PLS-1 mode. In order to select the number of factors, a cross-validation method, leaving out one sample at a time, was used. Given the set of 35 calibration voltammograms, the PLS- 1 calibration on 34 calibration curves was performed and, using this calibration, the concentration of the compounds in the sample left out during calibration was predicted. This process was repeated 35 times until each calibration sample had been left out once. The concentration of each sample was then predicted and compared with the known concentration of the reference sample. The values of the root mean square error of calibration (RMSEC), which is an estimate of the absolute error of calibration and the values of the root mean square of the prediction
(RMSEP) and the squared correlation coefficients $\left(R^{2}\right)$, obtained when plots of actual versus measured (or predicted) predicted concentration are summarized in Table 3.

Results indicate that the optimized PLS-1 model allows us to assess simultaneously the IPT and ${ }^{\circ}$ Brix in grape juices.

\section{Conclusions}

A multisensor system was obtained by using screen-printed electrodes modified with carbon, platinum, gold, graphene, nickel oxide nanoparticles and Prussian blue, and covered with glucose oxidase or tyrosinase. The voltammetric responses toward glucose and catechol demonstrated that each screen-printed material shows a different electro-catalytic and electron mediator activity, producing a variety of responses. The electrochemical signals of musts consisted of complex voltammetric curves bearing information associated with the phenolic and sugar content of the grapes. In addition, the interactions between the electrode and the solution (i.e. the $\mathrm{pH}$ affecting the enzymatic activity, or the electrocatalytic activity of the modifiers) increased the cross-selectivity of the sensors.

A clear discrimination of the samples was attained by using the hybrid array formed by glucose oxidase and tyrosinase based sensors, combined with Principal Components Analysis.

The enhanced electrochemical reactivity of the M-GOX-SPE and M-Tyr-SPE electrodes (particularly when using Prussian Blue and nickel oxide nanoparticles), and the variety of responses obtained when electrodes were immersed in musts, make these modified screen-printed electrodes attractive to form an array of sensors.

The system developed showed almost the same discrimination capability as other arrays based on more complex sensors. Due to the limited repeatability of the serigraphied electrodes, each sensor could only be used to analyze one sample. However, the lower price, ease of use and portability of the modified screen-printed electrode system makes the bioelectronic tongue developed here a possible alternative tool to analyze simultaneously sugar and phenols in situ in the vineyard block. However, further work is needed to reduce the number of sensors, so the system could be used more easily in a non-laboratory environment. There is also a need to extend the study to grapes with different degree of maturity.

\section{Acknowledgments}

Financial support by the MINECO (grant CICYT-AGL2012-33535) and Junta de Castilla y León (VA-032U13) is gratefully 


\section{acknowledged. CMP would also like to thank the University of Valladolid for the grant (PIF-UVa).}

\section{References}

Apetrei, C. (2012). Novel method based on polypyrrole-modified sensors and emulsions for the evaluation of bitterness in extra virgin olive oils. Food Research International, 48, 673-680.

Apetrei, I. M., Rodríguez-Méndez, M. L., Apetrei, C., Nevares, I., Del Alamo, M., \& De Saja, J. A. (2012). Monitoring of evolution during red wine aging in oak barrels and alternative method by means of an electronic panel test. Food Research International, 45, 244-249.

Baldwin, E. A., Bai, J., Plotto, A., \& Dea, S. (2011). Electronic noses and tongues: applications for the food and pharmaceutical industries. Sensors, 11, 4744-4766.

Barroso, M. F., De los Santos Alvarez, N., Delerue-Matos, C., \& Oliveira, M. B. P. P. (2011). Towards a reliable technology for antioxidant capacity and oxidative damage valuation: electrochemical (bio)sensors. Biosensors and Bioelectronics, $30,1-12$.

Blouin, J., \& Guimberteau, G. (2000). Maturation et maturité des raisins. Bordeaux France: Éditions Féret.

Carralero Sanz, V., Mena, M. L., González-Cortés, A., Yáñez-Sedeño, P., \& Pingarrón, J. M. (2005). Development of a tyrosinase biosensor based on gold nanoparticles-modified glassy carbon electrodes: application to the measurement of a bioelectrochemical polyphenols index in wines. Analytica Chimica Acta, 528, 1-8.

Cetó, X., Apetrei, C., Del Valle, M., \& Rodríguez-Méndez, M. L. (2014). Evaluation of red wines antioxidant capacity by means of a voltammetric e-tongue with an optimized sensor array. Electrochimica Acta, 120, 180-186.

Cetó, X., Gutiérrez, J. M., Gutiérrez, M., Céspedes, F., Capdevila, J., Mínguez, S., et al. (2012). Determination of total polyphenol index in wines employing a voltammetric electronic tongue. Analytica Chimica Acta, 732, 172-179.

Chaubey, A., \& Malhotra, B. D. (2002). Mediated biosensors. Biosensors and Bioelectronics, 17, 441-456.

Gay, M., Apetrei, C., Nevares, I., Del Alamo, M., Zurro, J., Prieto, N., et al. (2010). Application of an electronic tongue to study the effect of the use of pieces of wood and micro-oxygenation in the aging of red wine. Electrochimica Acta, 55, 6782-6788.

Ghozlen, N. B., Cerovic, Z. G., Germain, C., Toutain, S., \& Latouche, G. (2010). Nondestructive optical monitoring of grape maturation by proximal sensing. Sensors, 10, 10040-10068.

Gil-Sánchez, L., Soto, J., Martínez-Máñez, R., Garcia-Breijo, E., Ibáñez, J., \& Llobet, E. (2011). A novel humid electronic nose combined with an electronic tongue for assessing deterioration of wine. Sensors and Actuators A, 171, 152-158.

Gutiérrez-Osuna, R., \& Nagle, H. T. (1999). A method for evaluating datapreprocessing techniques for odor classification with an array of gas sensors. IEEE Transactions on Systems Man and Cybernetics Part B-Cybernetics, 29, 626-632.

Gutiérrez, M., Domingo, C., Vila-Planas, J., Ipatov, A., Capdevila, F., Demming, S., et al. (2011). Hybrid electronic tongue for the characterization and quantification of grape variety in red wines. Sensors and Actuators B, 156, 695-702.

Gutiérrez, J. M., Moreno-Barón, L., Pividori, M. I., Alegret, S., \& Del Valle, M. (2010) A voltammetric electronic tongue made of modified epoxy-graphite electrodes for the qualitative analysis of wine. Microchimica Acta, 169, 261-268.

Haddi, Z., Mabrouk, S., Bougrini, M., Tahri, K., Sghaier, K., Barhoumi, H., et al. (2014). E-Nose and e-Tongue combination for improved recognition of fruit juice samples. Food Chemistry, 150, 246-253.

Herrera, J., Guesalaga, A., \& Agosin, E. (2003). Shortwave-near infrared spectroscopy for non-destructive determination of maturity of wine grapes. Measurement Science and Technology, 14, 689-697.

Matemadombo, F., Apetrei, C., Nyokong, T., Rodríguez-Méndez, M. L., \& De Saja, J. A. (2012). Comparison of carbon screen printed and disk electrodes in the detection of antioxidants using CoPc derivatives. Sensors and Actuators $B$ 166-167, 457-466.
Medina-Plaza, C., De Saja, J. A., \& Rodríguez-Méndez, M. L. (2014). Bioelectronic tongue based on lipidic nanostructured layers containing phenol oxidases and lutetium bisphthalocyanine for the analysis of grapes. Biosensors and Bioelectronics, 57, 276-283.

Medina-Plaza, C., Revilla, G., Muñoz, R., Fernández-Escudero, J. A., Barajas, E., Medrano, G., et al. (2014). Electronic tongue formed by sensors and biosensors containing phthalocyanines as electron mediators. Application to the analysis of red grapes. Journal of Porphyrins and Phthalocyanines, 18, 76-86.

Metters, J. P., Kadara, R. O., \& Banks, C. E. (2011). New directions in screen printed electroanalytical sensors: an overview of recent developments. Analyst, 136, 1067-1076.

Moreno i Codinachs, L., Kloock, J. P., Schöning, M. J., Baldi, A., Ipatov, A., Bratov, A., et al. (2008). Electronic integrated multisensor tongue applied to grape juice and wine analysis. Analyst, 133, 1440-1448.

OIV. (2013). Compendium of international methods of analysis of wines and musts (Vol. 2). Paris, France: Organisation Internationale de la Vigne et du Vin.

Parra, V., Hernando, T., Rodríguez-Méndez, M. L., \& De Saja, J. A. (2004). Electrochemical sensor array made from bisphthalocyanine modified carbon paste electrodes for discrimination of red wines. Electrochimica Acta, 49, 5177-5185.

Prieto, N., Oliveri, P. Leardi, R. Gay, M., Apetrei, C., Rodriguez-Mendez, M. L., et al. (2013). Application of a GA-PLS strategy for variable reduction of electronic tongue signals. Sensors and Actuators B, 183, 52-57.

Rahman, Md. M., Ahammad, A. J. S., Jin, J., Ahn, S. J., \& Lee, J. J. (2010). A comprehensive review of glucose biosensors based on nanostructured metaloxides. Sensors, 10, 4855-4886.

Ricci, F., Amine, A., Palleschi, G., \& Moscone, D. (2003). Prussian Blue based screen printed biosensors with improved characteristics of long-term lifetime and $\mathrm{pH}$ stability. Biosensors and Bioelectronics, 18, 165-174.

Riul, A., Jr., Dantas, C. A. R., Miyazaki, C. M., \& Oliveira, O. N., Jr. (2010). Recent advances in electronic tongues. Analyst, 135, 2481-2495.

Rodríguez-Méndez, M. L., Apetrei, C., Gay, M., Medina-Plaza, C., De Saja, J. A. Vidal, S., et al. (2014). Evaluation of oxygen exposure levels and polyphenolic content of red wines using an electronic panel formed by an electronic nose and an electronic tongue. Food Chemistry, 155, 91-97.

Rodríguez-Méndez, M. L., Medina, C., De Saja, J. A., Apetrei, C., \& Muñoz, R. (2012). Sensor arrays based on phthalocyanines: new developments on nanostructured and biomimetic electrochemical sensors. In L. Lvova, D. Kirsanov, C. Di Natale, \& A. Legin (Eds.), Multisensor systems for chemical analysis: Materials and sensors (pp. 70-109). Singapore: Pan Stanford Publishing, Inc.

Sghaier, K., Barhoumi, H., Maaref, A., Siadat, M., \& Jaffrezic-Renault, N. (2009). Classification and discrimination of different Tunisian water samples using an electronic tongue. Sensor Letters, 7, 683-688.

Sliwinska, M., Wisniewska, P., Dymerski, T., Namiesnik, J., \& Wardencki, W. (2014). Food analysis using artificial senses. Journal of Agricultural and Food Chemistry, 62, 1423-1448.

Smyth, H., \& Cozzolino, D. (2013). Instrumental methods (spectroscopy, electronic nose, and tongue) as tools to predict taste and aroma in beverages: advantages and limitations. Chemical Reviews, 113, 1429-1440.

To Thi Kim, L., Gabrielli, C., Perrot, H., Garcia-Jareno, J., \& Vicente, F. (2012). Redox switching of Prussian blue thin films investigated by ac-electrogravimetry. Electrochimica Acta, 84, 35-48.

Valentini, F., Carbone, M., \& Palleschi, G. (2013). Graphene oxide nanoribbons (GNO), reduced graphene nanoribbons (GNR), and multi-layers of oxidized graphene functionalized with ionic liquids (GO-IL) for assembly of miniaturized electrochemical devices. Analytical and Bioanalytical Chemistry, 405, 3449-3474.

Wang, J. (2008). Electrochemical glucose biosensors. Chemical Reviews, 108, $814-825$.

www.dropsens.com (consulted for the last time on February the 18th, 2015).

Zeravik, J., Hlavacek, A., Lacina, K., \& Skládal, P. (2009). State of the art in the field of electronic and bioelectronic tongues - towards the analysis of wines. Electroanalysis, 21, 2509-2520. 